\title{
Permanent Magnet for the Beam Profile Monitor
}

\author{
V. Kashikhin, B. Brown, D. Morris, W. Robotham, M. Tartaglia, R. Thurman-Keup, G. Velev, J. Volk, J. Zagel
}

\begin{abstract}
The ionization beam profile monitor system for the Main Injector Ring is under construction at Fermilab. The beam profile detector unit is installed inside the main magnet gap. The magnet has a novel configuration previously used for this type of application in the Main Injector. However this magnet is far more compact with a higher quality field. Most flux from the main gap returns symmetrically along the beam pipe through two side gaps. It provides nearly full compensation to yield integrated magnetic field close to zero, and helps eliminate distortions of the circulating proton beam. The permanent magnet poles are assembled from SmCo5 bricks (0.5"x1"x2") which have a good thermal stability, and a reasonable cost. Further integrated field reduction is obtained by the use of a ferromagnetic plate which shunts the main gap. The plate position and flux shunting are adjusted in conjunction with magnetic measurements. Three permanent magnets were successfully fabricated and measured. Results of the magnet design, 3D FEA analysis, and magnetic measurements by the rotational coil and the 3D Hall probe will be presented.
\end{abstract}

Index Terms - Permanent Magnet, Ionization Profile Monitor, Design, Fabrication, Magnetic Measurements.

\section{INTRODUCTION}

$I^{-}$ ONIZATION beam profile monitors (IPM) are successfully

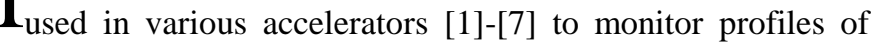
charged particles beams. One of the main parts of this system is a dipole magnet which generates the magnetic field in the detector area. The detector is mounted inside the magnet gap. Various types of magnets were used: a single electromagnet [5], two electromagnets of opposed polarity [6], two pole permanent magnet [3], three pole permanent magnet [8]. The permanent magnet approach has valuable advantages: no power supply, do not need water for coil cooling, properly calibrated does not produce circulating beam distortion, easy to install. In this paper we describe the permanent magnet system, including design and magnetic measurements and method of magnet fine tuning.

\section{Permanent Magnet Design}

Two configurations of the IPM magnet were investigated, having two or three air gaps. A two gap magnet could be made more compact but the magnet with three gaps produces less distortion for the circulating beam and was chosen as a

Manuscript received July 15, 2013. This work was supported in part by Fermi Research Alliance, LLC, under contract No. DE-AC02-07CH11359 with the U.S. Department of Energy.

V. S. Kashikhin\#, B. Brown, D. Morris, W. Robotham, M. Tartaglia, R. Thurman-Keup, G. Velev, J. Volk, J. Zagel are with Fermi National Accelerator Laboratory, Batavia, IL 60510 USA, corr. author \# (phone: 630840-2899; fax: 630-840-9999; e-mail: kash@fnal.gov). baseline design. For the permanent magnet material $\mathrm{SmCo}_{5}$ was chosen [9]. This type of material has good temperature stability and relatively low cost. Nevertheless, the cost of permanent magnet material is the main magnet cost driver. A permanent magnet design is a rather challenging task if it is combined with an iron yoke [10]. The main goal for the design is to achieve the specified magnet strength and field quality with the minimum permanent magnet material volume, to minimize the magnet cost. Before starting a time consuming 3D FEM permanent magnet analysis, an initial configuration was estimated by using Maxwell's equations

$$
\begin{gathered}
\oint \boldsymbol{H} d \boldsymbol{l}=0 \\
\iint \boldsymbol{B} d s=0
\end{gathered}
$$

and the relation between the flux density $\boldsymbol{B}$, Field strength $\boldsymbol{H}$, and the magnetization vector $\boldsymbol{M}$ of the permanent magnet:

$$
\boldsymbol{B}=\mu_{0} \boldsymbol{H}+\boldsymbol{M}
$$

Equations (1) - (3) for the IPM magnet with three gaps (See Fig. 1) could be presented in the form of magneto-motive force (4) and flux density (5) balances, where $m, 0, s$ indicate the magnetic, air, and shunt regions:

$$
\begin{aligned}
& H_{m} l_{m}+H_{0} \delta_{0}+H_{s} \delta_{s}=0 \\
& B_{m} A_{m}=B_{0} A_{0}=2 B_{s} A_{s}
\end{aligned}
$$

The permanent magnet (PM) properties can be presented using coercive force $H_{c}$ and remnant flux density $B_{r}$ using (3):

$$
B_{m}=B_{r}\left(1-\frac{H_{m}}{H_{c}}\right)
$$

The solution of (4) - (6) gives the expression for the magnet field in the IPM detector area:

$$
B_{0}=\frac{\mu_{0} H_{c} l_{m}}{\frac{\mu_{0} H_{c} A_{0} l_{m}}{B_{r} A_{m}}-\delta_{0}-\frac{A_{0} \delta_{s}}{2 A_{s}}}
$$

where A - effective flux areas of magnet air gaps and permanent magnet area, $l_{m}$ - the permanent magnet length along the magnetization vector $\mathbf{M}$.

The magnet pole area for the iron dominated magnets defined by a specified magnetic field quality of $1 \%$ or better in the detector volume, a cube with $100 \mathrm{~mm}$ sides. Also, the integrated magnetic field along the beam path should be zero to eliminate distortion of the circulating in the Main Injector beam: 


$$
\int_{-\infty}^{\infty} B_{y} d x=0
$$

This integral should be less than $10 \mathrm{G}-\mathrm{m}$ which is a very strong requirement for the IPM magnet design and fabrication.

\section{A. The choice of Magnet Configuration}

The magnet consists of the main air gap for the detector region and two side gaps for the circulating beam (See Fig. 1 and Fig. 2). The magnet main pole tips have shims. PM magnetic bricks are placed between pole tip and the yoke. Both pole assemblies are supported by aluminum $\mathrm{C}$-shaped spacers.

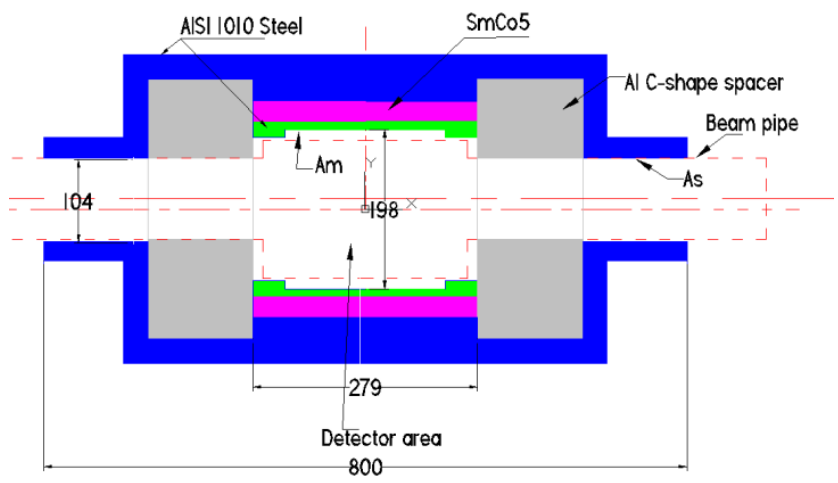

Fig. 1. IPM magnet configuration (front view), dimensions are in $\mathrm{mm}$.

The magnet detector area is much larger than the beam pipe between side poles. This is why the width of side poles was reduced (See Fig. 3).

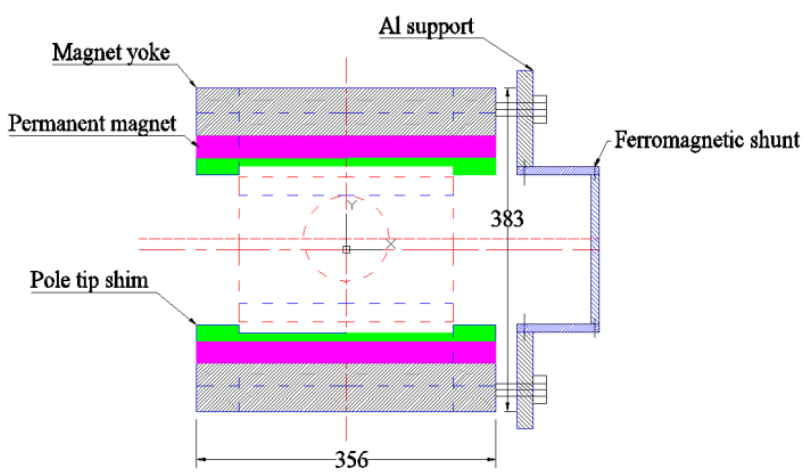

Fig. 2. IPM magnet configuration (side view), dimensions are in $\mathrm{mm}$.

The ferromagnetic shunt is shown in Fig. 2: the gap between $\mathrm{PM}$ and shunt can be adjusted in the range of $5 \mathrm{~mm}-30 \mathrm{~mm}$. The smaller this gap the larger is the shunting effect and lower is the central part of integrated field relative to side gaps.

After the first IPM magnet simulation, analytic formulas (4) - (7) were used to calculate the thickness and area of PM material needed to obtain $0.1 \mathrm{~T}$ field in the main gap. The PM material properties for $\mathrm{S} 2418$ [9] are: $B_{r}=1.0 \mathrm{~T}, \mu_{0} H_{c}=$ $0.94 \mathrm{~T}$. Flux efficient areas $\mathrm{A}_{0}$ and $\mathrm{A}_{\mathrm{s}}$ including fringe fields were taken from the first 3D simulation with $l_{m}=0.0127 \mathrm{~m}$ (one PM brick thickness). Using analytic formulas the PM dimensions to generate the specified $0.1 \mathrm{~T}$ center field were calculated: $l_{m}=0.0254 \mathrm{~m}$ (two PM bricks thicknesses) and $\mathrm{A}_{\mathrm{m}}$ $=0.1 \mathrm{~m}^{2}$.

\section{B. IPM Magnet 3D Simulations}

The IPM magnet has a complicated 3D configuration and was simulated by OPERA3d TOSCA code. The main goal for simulations was to obtain the $0.1 \mathrm{~T}$ magnetic field in the detector area with the field quality achievable given dimensional constraints (distances to tunnel ceiling, wall, available slot length), and zero integrated field along the beam orbit. This was achieved by proper main pole shimming (see Fig. 1) to improve the field homogenety in the main gap. The first run of simulations showed the integrated field to be far from zero because the main poles have large fringe fields on sides, which substantially reduces the flux and integrated field under side poles. To eliminate this effect the ferromagnetic shunt was introduced, which reduces the part of the integrated field between main poles relative to side poles. Because of PM brick magnetization and uncertainty in magnet yoke magnetic properties, combined with the strong limit of $10 \mathrm{G}-\mathrm{m}$ for the integrated field, the shunt was designed to be movable.

The simulated magnet geometry and the flux density distribution is shown in Fig. 3.

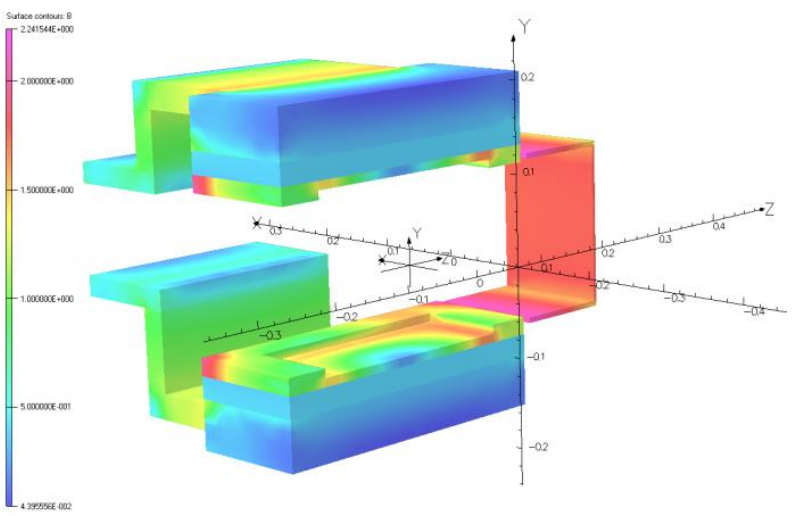

Fig. 3. IPM magnet flux density distribution (half magnet is shown).

The relatively thin shunt chosen is saturated $(\mathrm{B}>2 \mathrm{~T})$ even at $25 \mathrm{~mm}$ distance from the yoke. Fig. 4 shows that pole tip shims improve the detector area field homogeneity to $1 \%$.

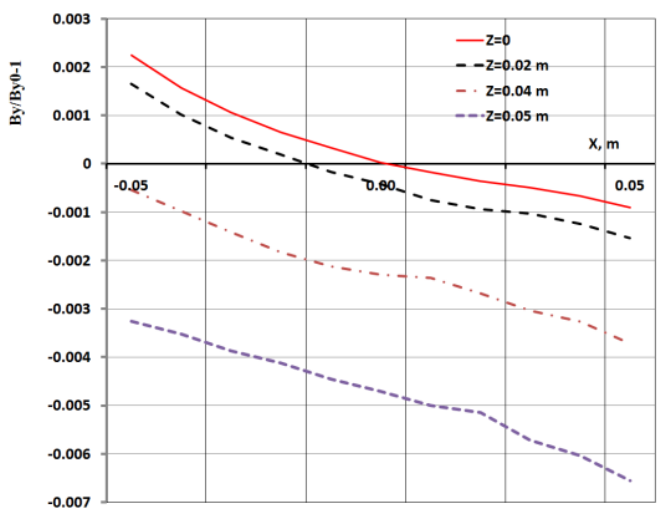

Fig. 4. Magnetic field homogeneity in the magnet middle plane of the detector area. 
It should be noted that the main pole integrated field is +338 G-m as shown in Fig. 5 and obtaining the differential integrated field of several G-m is not a trivial task.

The ability of the shunt to provide the wide variation between the positive and negative parts of the integrated field was verified, as shown in Fig. 6.

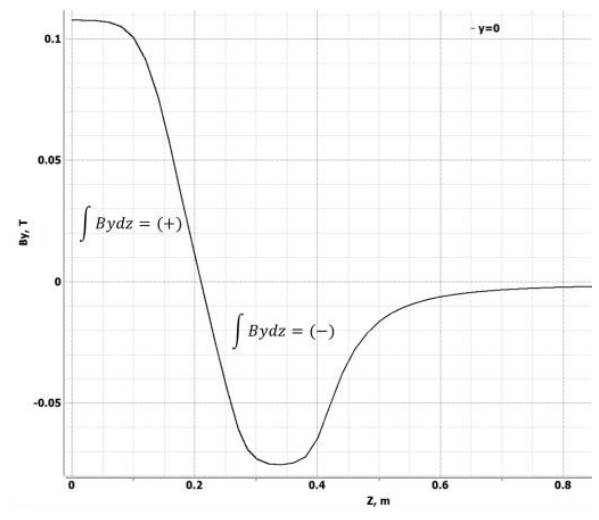

Fig. 5. Vertical field component distribution along the beam path.

Several variants of the magnet with different shunt positions were calculated. The strong linear shunting effect was observed (See Fig. 6). The integrated field variation was in the range of $20 \mathrm{G}-\mathrm{m}$. It should be noted that the main pole integrated field is $338 \mathrm{G}-\mathrm{m}$ and obtaining the differential integrated field of several G-m is not a trivial task. Fig. 6 shows the variation of integrated field versus shunt position, with respect to the yoke steel.

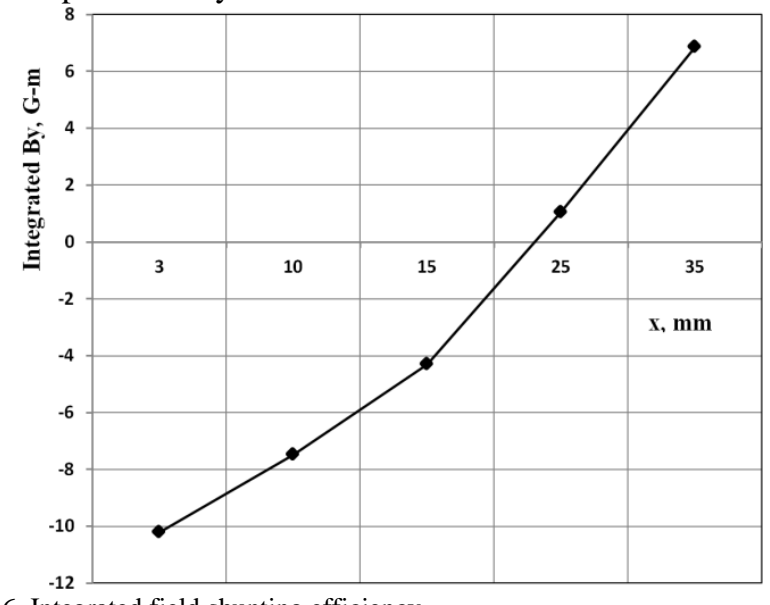

Fig. 6. Integrated field shunting efficiency.

The zero integrated field could be obtained at the shunt distance of $15 \mathrm{~mm}$ (0.59") from the pole tip. This result was confirmed by magnetic measurements (See Fig. 9).

\section{IPM Magnet Fabrication}

Three IPM magnets were assembled by HI-TECH Manufacturing [10] using FNAL design drawings. The pole permanent magnet block was assembled from the magnetized $\mathrm{SmCo}_{5}$ bricks with dimensions $2 \times 1 \times 0.5$ inches. The direction of magnetization was along the 0.5 inch dimension. Two layers of these bricks were placed to form one inch of pole block thickness. One of the issues during assembly was transverse forces between bricks, in spite of large vertical force between the low carbon steel yoke and PM material. Even a small gap between PM bricks is capable of substantially degrading the PM material efficiency because of parasitic fluxes between bricks. So, stainless steel nonmagnetic side plates were added to tighten the assembly in the transverse direction (See Fig. 7). After the first run of magnetic measurements, the movable ferromagnetic shunt was found to give a good shunting effect.

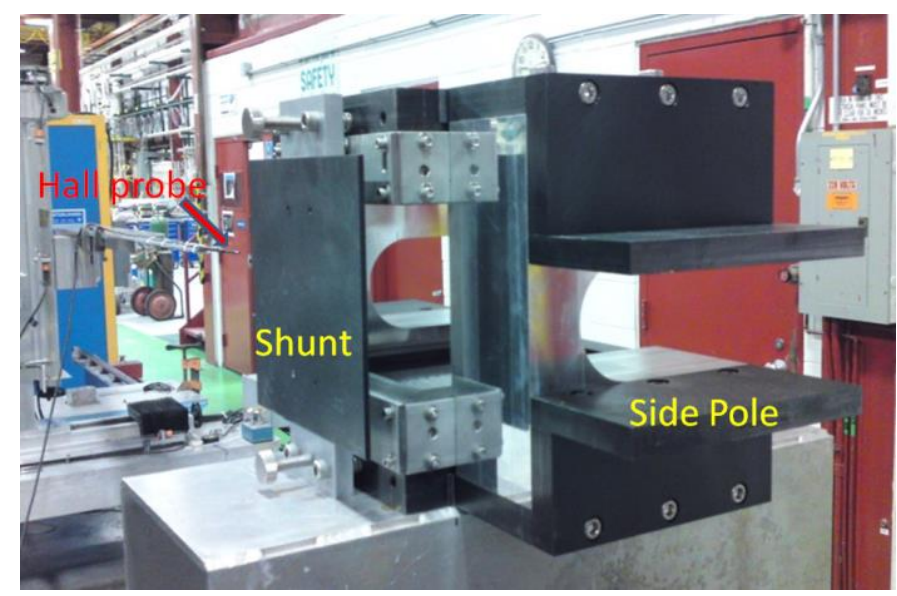

Fig. 7. The IPM magnet with the initial configuration.

The second run of magnetic measurements was with a modified shunt (See Fig. 8). This shunt improved the field homogeneity in the detector area because it has the vertical plate further from the main magnet gap.

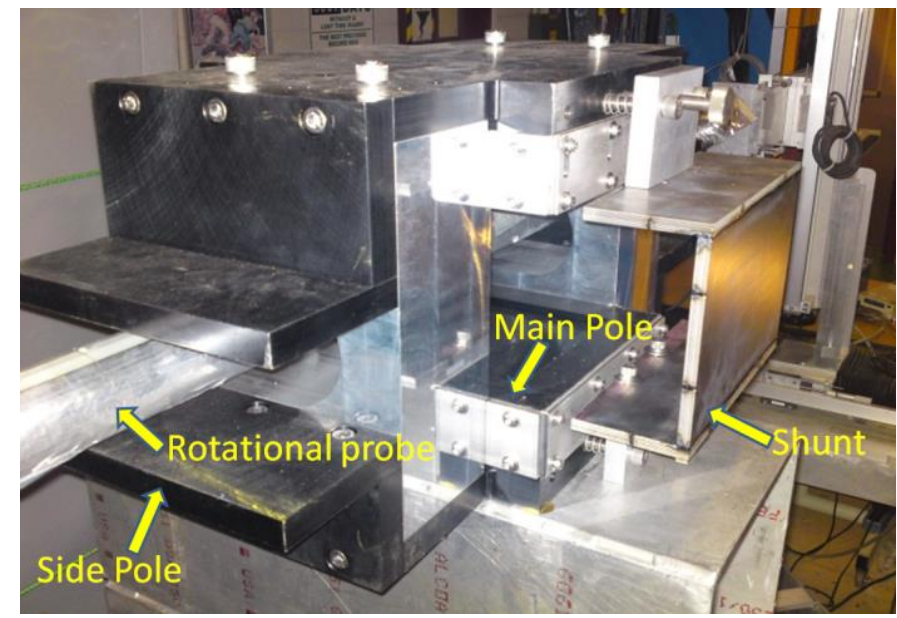

Fig. 8. Updated IPM magnet with the new ferromagnetic shunt.

\section{MAGNETIC MEASUREMENTS}

Magnetic measurements were made during the process of adjusting the shunt plates for each of the IPM magnets. First, the dipole field integral was measured as a function of the shunt plate distance from the magnet body. A long rotating 
harmonic coil probe of radius 1.29" was used for this measurement; the Main Injector beam tube is 2" in radius, so this probe sampled somewhat less than the full field seen by beam. For one of the three magnets, the field integral was measured also with the probe offset by 1.5 " in the horizontal direction, and determined the integral was identical to the onaxis field. Fig. 9 shows the dependence of the field integral with the modified shunt plate position, which is very similar for all three magnets.

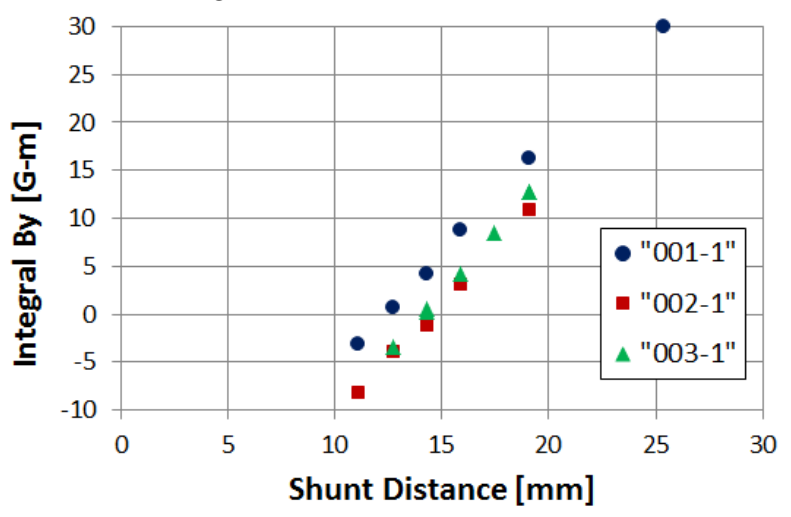

Fig. 9. Dipole field integral as a function of shunt distance from the magnet body (measured from stainless steel side plate, not steel yoke).

To determine the baseline strength, magnet 002-1 was first measured without a shunt plate: the result was $60 \mathrm{G}-\mathrm{m}$, which would correspond to a shunt distance of $40 \mathrm{~mm}$ (i.e., beyond this distance, the shunt has no influence on the field). The shunt plate positions were fixed in the position of minimum field integral, which was about $1.0 \mathrm{G}-\mathrm{m}$, or less, for all three magnets.

After the shunt adjustment, a profile of the (vertical) dipole field along the magnet (beam) axis was measured using a (1-D Group3) Hall probe for the first magnet. This confirmed that the general field shape agreed with that predicted by the model, shown in Fig. 5.

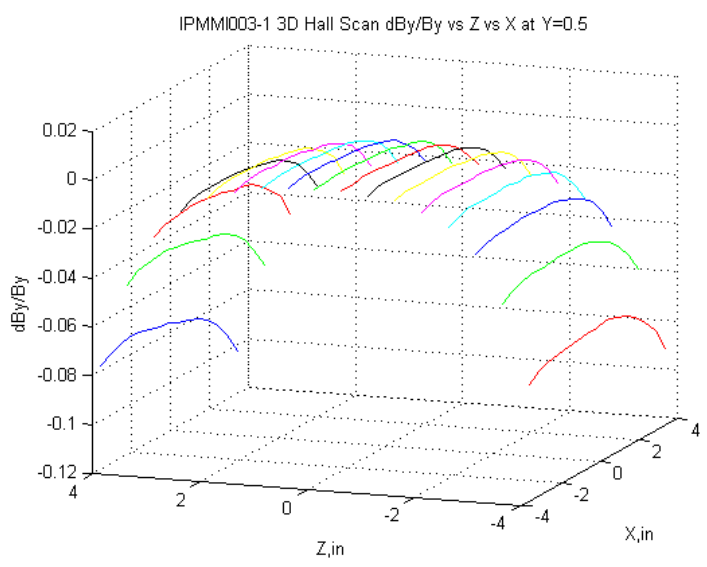

Fig. 10. Fractional deviation of By from value at the geometric center, as a function of $\mathrm{X}$ and $\mathrm{Z}$ at the beam axis vertical position.

Finally, a precision field map of the body field was made using a 3-D Senis $2 \mathrm{~T}$ Hall probe. Points were taken with 0.5 " spacing on a grid from -3.5 " to 3.5 " in X (horizontal), -2 " to 2 " in Y (vertical), and -4 " to 4 " in $\mathrm{Z}$ (beam direction). The grid was centered on the magnet geometrical center, which is lower by 0.5 " from the beam axis. The "uniform field region" for the profile monitor corresponds to a 4" cube centered on the beam axis, so this scan extends well beyond the needed volume. The measured shapes for $B_{x}, B_{y}$, and $B_{z}$ are virtually indistinguishable between the three magnets. Fig. 11 shows the dipole field uniformity $\left(\Delta \mathrm{B}_{\mathrm{y}} / \mathrm{B}_{\mathrm{y} 0}\right)$ in the $\mathrm{X}-\mathrm{Z}$ plane at $\mathrm{Y}=0.5$ " (the beam axis). Positive $\mathrm{X}$ is towards the shunt plate. The central dipole strengths $\mathrm{B}_{\mathrm{y} 0}$ were very similar: $0.1083 \mathrm{~T}$ for magnets 002 and 003 , and 0.1078 for magnet 001 .

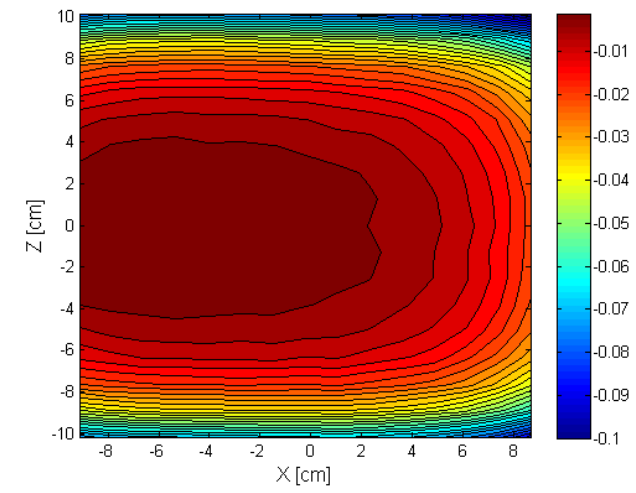

Fig. 11. Alternate figure showing $\Delta \mathrm{By} / \mathrm{By}_{0}$ contours; the variation with in the $\pm 5 \mathrm{~cm}$ uniform field region in $\mathrm{X}-\mathrm{Z}$ is within $1 \%$

\section{CONCLUSION}

Three IPM magnets were designed and tested at Fermilab. The design used a novel approach for PM magnets: a combination of analytical formulas with $3 \mathrm{D}$ magnetic field simulations. It allowed to obtain better than $1 \%$ uniformity, and agreement, between the calculated and measured central magnetic field. A new shunting approach for IPM magnet shimming was implemented, which enabled reducing the integrated field to a previously unachieved value, $<1.0 \mathrm{G}-\mathrm{m}$.

\section{REFERENCES}

[1] W. H. DeLuca, "Beam Detection Using Residual Gaslonization," IEEE Trans. Nuclear Science, NS-16, 1969, p. 813.

[2] J. R. Zagel, et al., "Improvements to the Fermilab Ionization Profile Monitor Systems," Particle Accelerator Conference, PAC 99, March 1999, pp. 2164-2166.

[3] W. C. Sellyey, J. D. Gilpatrick, "A Compact Residual Gas Ionization Profile Monitor (RGIPM) System," Particle Accelerator Conference, PAC 99, March 1999, pp. 2152-2154.

[4] P. Cameron, et al., "The RHIC Ionization Beam Profile Monitor," Particle Accelerator Conference, PAC 99, March 1999, pp. 2114-2116.

[5] Y. Sato, et al., "Development of Residual Gas Ionization Profile Monitor for High Intensity Proton Beams," IEEE Nuclear Science Symposium Conference, N22-2, 2005, pp. 1043-1046.

[6] A. Jansson, et al., "'An Ionization Profile Monitor for the Tevatron," Particle Accelerator Conference, PAC 2005, Knoxville, USA, 2005.

[7] A. Jansson, et al., "'Tevatron Ionization Profile Monitoring," European Particle Accelerator Conference, EPAC 2006, Edinburgh, UK, 2006.

[8] J. R. Zagel et al., "Permanent Magnet Ion Profile Monitor at the Fermilab Main Injector," Particle Accelerator Conference, PAC 2001, Chicago, 2001, pp. 1303-1305.

[9] "Dexter Magnetic Technologies," https://www.dextermag. com/S2418.

[10] P. Campbell, "Permanent Magnet Materials and their Application," Cambrige University Press, 1994, p. 207.

[11] HI-TECH Manufacturing LLC, http://www.hi-tech-mfg.com/. 